Background National Planning Department is the central organization granting project approvals in the government sector in Sri Lanka. As a prerequisite for implement a project, an approval is mandatory. This entire process was paper-based and possessed several limitations resulting in stagnations and rejections. Four major gaps identified in the current process: insufficient knowledge and experience of the personnel in project formulation and submission, unavailability of guiding materials in native languages, absence of project appraisal mechanism and lack of feedbacks. To address these issues, a set of interventions designed and implemented.

Methods Project was carried out in three phases at the decentralized health management units in Northern Province of Sri Lanka.

Phase I, a preintervention arm involved evaluation of current proposal statuses.

Phase II consisted design and implementation of interventions. Upon identifying gaps, an online proposal feedback portal that used colour coding and provided real-time feedbacks was developed. Health planning unit staff were trained through workshops to develop project proposals and get feedbacks via an online portal. A project appraisal committee also formed and guiding documents translated into native languages. Phase II spanned across three months.

Phase III comprised of post-intervention result assessment. Findings Post-interventional analysis indicates that all major gaps are bridged.

Evaluation of submitted and approved project proposals revealed a post-intervention approval rate of $60-100 \%$ as compared to pre-intervention which was $40-50 \%$.

Post-intervention mechanisms were rated more favorably than pre-intervention, including convenience to obtain feedback (mean 2.0-4.29) and were statistically significant.

Impact This research project is bringing an impact on Health Care System by ensuring the timely implementation of the projects without delay. It prevents cost, quality and time overruns.

\section{HEALTH WORKFORCE AND HOSPITAL CAPACITY MANAGEMENT IN THE CONTEXT OF COVID-19 PANDEMIC RESPONSE}

${ }^{1}$ Santoshi Balkrishna Sinai Malkarnekar, ${ }^{2}$ Priyanka suresh. ${ }^{1}$ Medical Superintendent, HAL Hospital, Bangalore, India; ${ }^{2}$ Medical officer, HAL Hospital , Bangalore , India

\subsection{6/leader-2021-FMLM.26}

Aims a) Health workforce and hospital capacity building in the covid-19 pandemic.

Challenges a) Lack of adequate staff capacity including ICU capable staff b) Need for real time re-designs of models for COVID-19 patients, without affecting essential services.

Intervention 1) Measures taken to improve staff levels:

Recruited and trained doctors and nurses from other specialties. Temporary re-deployment of clinicians of various specialties. New graduates were mentored and on-the-job training for generalist workers provided.

2) Measures taken to increase hospital capacity:

Reduced elective medical care Altered and re-purposed clinic and underused spaces for COVID-19 services.Home-based care for COVID-19 patients with mild or moderate symptoms.

3) Additional strategies :
Developed COVID-19 pandemic preparedness committee. Nodal officer nominated to liaise with government authorities. Rapid procurement of ventilators, oxygen concentrators and respirators for health care workers. Telemedicine platforms for clinical advice to patients.

Impact Expansion of staff and bed capacity, ensured that COVID-19 and non COVID-19 emergencies were treated adequately.

Conclusion Health care organizations will be challenged to adjust capacity, redesign care and redeploy staff in a pandemic. Building health workforce capacity, will support health systems in the immediate response and also form a foundation for global health security in future.

\section{HEALTHCARE IN THE TIME OF A PANDEMIC AND BEYOND: THE INNOVATIVE LARGE-SCALE AND INTEGRATED SAUDI NATIONAL HEALTH COMMAND CENTRE}

${ }^{1}$ Muaddi Alharbi* ${ }^{2}$ Mohammmed Senitan, ${ }^{3}$ Tim Ohanlon, ${ }^{4}$ Sidney Smith ${ }^{5}$ Dalia Mominkhan, ${ }^{1}$ Sarah Alqahtani, ${ }^{6}$ Mohammed Alabdulaali M. ${ }^{1}$ The Studies and Consulting Office at the Assistant Minister of Health Ministry of Health, Riyadh, Saudi Arabia; ${ }^{2}$ Faculty of Health Sciences, Saudi Electronic University, Riyadh, Saudi Arabia; ${ }^{3}$ Ascend Advanced Healthcare Solutions, Riyadh, Saudi Arabia; ${ }^{4}$ Medical Faculty, Division of Cardiology, University of North Carolina, US; ${ }^{5}$ National Health Command Centre, Riyadh, Saudi Arabia; ${ }^{6}$ Assistant Minister of Health Ministry of Health, Riyadh, Saudi Arabia

\subsection{6/leader-2021-FMLM.27}

Background The increasing frequency of pandemics, and costs of healthcare services requires integrative, efficient and effective health systems.

Aim Describe the framework and distinctive outcomes of the Saudi National Health Command Centre (NHCC). Method: A review was performed to describe the system-based engineering approach utilised to design the Saudi NHCC. This smart centre creates a new model of care delivery which impacts clinical and operational indicators by adopting integrative and interdisciplinary methods to analyse, disseminate, manage, and measure outcomes.

Results The NHCC is structured into four main departments with data integration and real-time data visualisation to allow for rapid assessment of available resources. It's organised to support technical incubators and empower several initiative. This enhanced the proactive capacity management in the centre and collectively contributed to several favourable outcomes. These included rapidly deploying medical staff and mechanical ventilators during its response to coronavirus disease (COVID-19); bending the curve early of the first wave of COVID-19 resulting in a low mortality rate $(<2 \%)$; and reducing ICU lengths of stay by $10 \%$, average lead-time of the supply chain from 60 days to 25 days, and surgery waiting times. It also supported the increment bed capacity from 6,000 to 10,400 , and maintaining the percentage of patients receiving care within 4 hours in emergency departments above $85 \%$.

Conclusion The NHCC replaces the traditional reliance on the subjectivity of information-based processes with actionable data, which helps building fairer systems to tackle structural inequalities in healthcare access and outcomes, eliminating waste, and allocating resources more effectively and efficiently. Importantly, a command centre to healthcare design and delivery creates synergy between people, processes, and technology 